\title{
Hemoglobin concentration is associated with self-reported disability and reduced physical performance in a community dwelling population of nonagenarians: the Mugello Study
}

\author{
Francesca Cecchi $^{1} \cdot$ Silvia Pancani $^{1} \cdot$ Federica Vannetti $^{1} \cdot$ Roberta Boni $^{1}$. \\ Chiara Castagnoli $^{1}$ - Anita Paperini ${ }^{1} \cdot$ Guido Pasquini $^{1} \cdot$ Francesco Sofi $^{1,2}$. \\ Raffaele Molino-Lova ${ }^{1}$ Claudio Macchi ${ }^{1,2}$ - Mugello Study Working Group ${ }^{3,4}$
}

Received: 7 August 2017 / Accepted: 14 October 2017 / Published online: 25 October 2017

(C) The Author(s) 2017. This article is an open access publication

\begin{abstract}
People aged 90 and older represent a fast-growing population segment who deserve specific attention and research. Aging is associated with a progressive decrease in hemoglobin concentration, which predicts adverse outcome, such as mortality, morbidity, frailty and disability. Whether this association is independent from increased prevalence of comorbidity, causing both anemia and reduced physical function is yet under debate. The aim of this study is to explore the relationship between hemoglobin concentration and self-reported disability and reduced physical performance in a community dwelling population of nonagenarians. Data presented were collected in the framework of the Mugello Study, a clinical epidemiologic survey of nonagenarians living in the Mugello area (Tuscany, Italy). 251 persons (177 women, age $93.2 \pm 3.3$ years; 74 men, age $92.2 \pm 2.5$ years) underwent a blood draw. Along with hemoglobin concentration, self-reported disability (basic and instrumental activities of daily living), physical performance (Short Physical Performance Battery), self-reported physical activity and muscular strength (handgrip measurement) were assessed. Covariates, inherent sociodemographic and health indicators and comorbidities were also included
\end{abstract}

The members of the Mugello Study Working Group are listed in Acknowledgements.

Silvia Pancani

silvia@pancani.eu

1 Don Carlo Gnocchi Foundation, IRCSS, Via di Scandicci, 269, 50143 Florence, Italy

2 Department of Experimental and Clinical Medicine, University of Florence, Florence, Italy

3 University of Florence, NEUROFARBA, Florence, Italy

4 Department of Health Science, University of Florence, Florence, Italy in the analysis. This study confirms that anemia is very common in the oldest old, with a significantly higher prevalence in males (50\% in men vs $24 \%$ in women). Multiple linear regression analysis, including all the comorbid conditions as confounding factors, shows that hemoglobin concentration is independently associated with handgrip strength, self-reported physical activity and disability in dressing, and taking a shower/bath. In conclusion, results from this study confirm that in the oldest old, low hemoglobin concentration is strongly associated with self-reported disability and decline of physical performance independent of comorbidity.

Keywords Hemoglobin - Anemia $\cdot$ Physical performance $\cdot$ Handgrip $\cdot$ Nonagenarians $\cdot$ Oldest old

\section{Introduction}

As the world population is constantly aging, the oldest old, often defined as those aged $85+$ or $90+$, present the largest increase in numbers [1]. The saying "the nineties are the new eighties," [2] well expresses the socio-epidemiological and clinical relevance of this trend. Addressing modifiable manifestations of aging that are relevant to function and quality of life is one of the main goals of geriatric medicine, and persons aged 90, besides being simply older, are a somewhat selected population who deserve specific attention and research [3,4]. Thus, the identification and treatment of those medical conditions that impact on function and quality of life in the oldest old becomes of critical importance for healthcare provision.

Aging is often associated with a progressive decrease in hemoglobin concentration and erythrocyte indices, due to several factors including decreased erythropoietin secretion, reduced hematopoietic reserve, reduced iron and vitamin 
intake, and morbid conditions such as chronic inflammation [3]. Although reports of incidence and prevalence of anemia in the elder population are rather discrepant, a steeper decrease of hemoglobin concentration values in the oldest old (age range $85+$ ) has been observed [5].

Anemia, or even mildly low hemoglobin concentration above the WHO threshold for anemia (i.e., $120 \mathrm{~g} / \mathrm{l}$ for women and $130 \mathrm{~g} / \mathrm{l}$ for men) consistently predict worse outcome, including mortality, hospitalization, and falls in the elderly population [5-9]. Recent studies also suggest an independent association in old age between anemia and frailty, and disability [10-13]. However, most of these studies include persons above 60 or 65 , while less is known about the oldest old. A paramount issue is whether adverse physical and functional outcomes in this specific share of the population are independently associated with reduced hemoglobin concentration, or rather, as suggested by a recent large Dutch study on the $85+$ population [14], they are mainly explained by highly prevalent comorbidity, causing both anemia and reduced physical function.

The Mugello Study [15] is a clinical epidemiologic survey of nonagenarians living in the Mugello area, north-eastward of Florence, Tuscany, Italy. This area is characterized by a higher percentage of nonagenarians with respect to the entire population, $1.76 \%$, compared to the Italian reference value, $1.14 \%$ (ISTAT-Data 2015).

The purpose of the present study is to investigate if and how hemoglobin concentration is related to a reduced physical performance and disability in a community dwelling population of nonagenarians.

\section{Methods}

\section{Participants}

The analysis presented in this paper is based upon data from the Mugello Study, a survey on nonagenarians living in the Mugello area, north-eastward of Florence, in Tuscany, Italy, whose detailed description is reported elsewhere [15]. Briefly, all community dwelling persons aged $90+$ living in the Mugello area who accepted to be enrolled, underwent a structured interview and medical visit, along with a series of instrumental assessments at their home. Blood samples were also collected to perform routine laboratory tests, and to create a biological bank with serum and plasma aliquots. The Mugello Study protocol, which complied with the principles of the Declaration of Helsinki on clinical research involving humans, was approved by the Ethics Committee of the Don Gnocchi Foundation, and the participants, or their legal representative, signed the informed consent form to be included in the study, and to undergo blood withdrawal.
Among the Mugello Study participants, 98 (74 F mean age $93.5 \pm 3.8-24 \mathrm{M}$ mean age $93.1 \pm 3.7$ ) subjects refused to provide a blood sample, and were thus excluded from this study, while 251 subjects ( $177 \mathrm{~F}$ mean age $93.2 \pm 3.3-74 \mathrm{M}$ mean age $92.2 \pm 2.5$ ) had completed data to be included in this analysis.

\section{Main variables}

Hemoglobin concentration, g/dl, was analyzed using the hematology analyzer Sysmex XT-1800 (Sysmex, Inc., Mundelein, IL). Hemoglobin concentration values above $16.5 \mathrm{~g} /$ $\mathrm{dl}$ (six cases) were excluded from further analyses since high blood viscosity was considered a sign of underlying serious clinical condition [3].

Disability was assessed according to the Katz questionnaire [16] concerning activities of daily life, six basic (eating, showering, dressing, transferring from bed to chair, using the toilet, and continence) and eight instrumental activities (shopping, doing light housework, preparing meals, managing money, using the telephone, taking medications, using transportation, doing laundry) [17]. The score for each activity ranged from 0 to 4; 0 no functional limitation and 4 total dependence. A score over or equal to 2 was assumed as an index of disability. Together with the dichotomic variable created for each activity, the sum (nADL) of reported basic disabilities (BADL) and instrumental disabilities (IADL) was retained for further analysis.

Physical performance was measured using the Short Physical Performance Battery (SPPB) [18], which assesses the walking speed, standing balance and ability to raise from a chair. The total score (SPS), developed by summing the partial scores of each task, ranges from 0 , in case a participant is not able to complete one test, to 12 , best performance.

Level of physical activity (SPA) was investigated and scored by administering a questionnaire to each nonagenarian. This questionnaire, which has been described elsewhere was modeled on the Harvard Alumni Questionnaire, and adapted for Italian people [15].

Muscle strength was evaluated by means of handgrip force measurement, expressed in $\mathrm{kg}$, using a hydraulic dynamometer (RO + TEN, Verano Brianza, Italy) [19]. Participants repeated the test twice for each hand, and the best value for each side was recorded. In this analysis, the mean value of the best right and left results was used.

In the analysis, covariates, inherent socio-demographics (age, sex) and health indicators were included. Body mass index was calculated as the ratio between weight $(\mathrm{kg})$ and the square of height $(\mathrm{m})$. Comorbidities, such as myocardial infarction, diabetes mellitus, cerebrovascular disease, peripheral artery disease, congestive heart failure, chronic obstructive pulmonary disease, chronic kidney disease, cancer, and gastric ulcer, also considered as dichotomous 
variables, were determined using an adjudication process that includes self-reported history, medical examination data, and medical records information [15].

Serum levels of high-sensitivity C-reactive protein (hsCRP) were measured according to WHO First International Reference Standard, with a sensitivity of $0.08 \mu \mathrm{g} / \mathrm{ml}$ using an enzyme-linked immunosorbent assay (ELISA), and a colorimetric competitive immunoassay that used purified protein and polyclonal anti-CRP antibodies; serum creatinine level was assessed using a standard creatinine Jaffe method (Roche Diagnostics, GmbH, Mannheim, Germany), which had an inter-assay coefficient of less than $2.5 \%$ [15].

\section{Statistics}

Data are reported as percentage or as mean \pm standard deviation. The analysis was performed using SPSS 23.0 software (IBM SPSS, Chicago, IL, USA).

Because of a high skewness some variables were transformed to approximate a normal distribution. A logarithmic transformation was applied to the variables $\mathrm{C}$-reactive protein and creatinine, while a square root transformation was applied to the variable handgrip.

The relationship between hemoglobin concentration and variables that describe disability and physical performance was investigated using a multiple linear or a logistic regression as appropriate, corrected by age and gender.

Similarly, multiple linear/logistic regression analyses were conducted to assess whether the effect of $\mathrm{HC}$ level on physical and performance parameters was still significant after adjustment for those comorbid conditions that were shown to be associated with hemoglobin concentration with a $p$ value at least $<0.05$.

\section{Results}

Participants' functional and physical characteristics are summarized in Table 1.

Although hemoglobin concentration data were substantially identical in men $(n=74$; mean HC $12.85 \pm 1.64)$ and women $(n=177$; mean HC $12.84 \pm 1.43)$, the prevalence of anemia according to the WHO different cut offs for men and women was much higher in men $(50 \%$ of men vs $24.3 \%$ in women), with an overall prevalence of $31.9 \%$. After adjusting for age and gender, associations between hemoglobin concentration and disability and physical performance are shown in Table 2. Handgrip strength, SPS score, nADL score and SPA are significantly associated with $\mathrm{HC}$ level, while there is no association BMI and hemoglobin concentration. When BADL and IADL activities are individually investigated, a significant association between hemoglobin concentration and dressing, taking a
Table 1 Participants' characteristics expressed as mean \pm SD or as a percentage as appropriate for continuous or ordinal variables

\begin{tabular}{ll}
\hline & Mean \pm SD (\%) \\
\hline Age (years) & $92.9 \pm 3.1$ \\
Gender (\%woman) & 71 \\
Hemoglobin (g/dl) & $12.8 \pm 1.5$ \\
Handgrip (kg) & $14.6 \pm 7.0$ \\
BMI (kg/m ${ }^{2}$ ) & $25.5 \pm 4.7$ \\
SPS (score) & $2.9 \pm 3.8$ \\
nADL (score) & $5.5 \pm 4.5$ \\
Score physical activity (score) & $0.7 \pm 0.9$ \\
BADL (\%Y) & \\
Eating & 7 \\
Dressing & 40 \\
Taking a shower & 63 \\
Using the toilet & 31 \\
Moving from chair to bed and vice versa & 29 \\
Incontinence & 62 \\
IADL (\% Y) & \\
Using the telephone & 29 \\
Shopping & 57 \\
Cooking & 39 \\
Doing light housework & 41 \\
Doing heavy housework & 68 \\
Doing laundry & 50 \\
Using public transport & 48 \\
Managing medication & 49 \\
Keeping track of finances & \\
Washing & 42 \\
Walking upstairs & \\
Move from one room to another & \\
Walking on a flat floor & \\
\hline
\end{tabular}

shower, doing laundry and using public transport activities is observed (Table 2). Comorbidities significantly associated with hemoglobin concentration are severe kidney disease and congestive heart failure (Table 3). Similarly, a significant association emerges between hemoglobin concentration and creatinine levels, while no association is observed with the C-reactive protein (Table 3 ).

When the multiple linear regression analysis was performed including all the comorbid conditions associated with hemoglobin concentration $(p<0.05$ in Table 3$)$ and the creatinine levels as confounding factors, hemoglobin concentration alone explains the $43 \%$ of the variance in the handgrip strength measured and shows a significant association with SPS, nADL and SPA scores (Table 4). The results of regression analysis for those BADL and IADL that showed a $p$ value $<0.05$ in Table 2 , adjusted for age, gender, comorbidities and creatinine indicate that as hemoglobin concentration increases, the odds of 
Table 2 Multiple linear and logistic regression model adjusted for age and sex showing the association between hemoglobin concentration and variables that describe physical performance and disability

\begin{tabular}{|c|c|c|c|c|}
\hline $\begin{array}{l}\text { Hemoglobin concentration }(\mathrm{g} / \mathrm{dl}) \text { adjusted for } \\
\text { age, sex }\end{array}$ & $\beta \pm \operatorname{SE}(\beta)$ & $R^{2}$ & OR & $p$ \\
\hline Handgrip_tr (kg) & $0.079(0.031)$ & 0.402 & & 0.010 \\
\hline BMI $\left(\mathrm{kg} / \mathrm{m}^{2}\right)$ & $-0.227(0.199)$ & 0.013 & & 0.253 \\
\hline Sps (score) & $0.361(0.158)$ & 0.097 & & 0.023 \\
\hline nADL (score) & $-0.384(0.193)$ & 0.176 & & 0.048 \\
\hline SPA (score) & $0.324(0.155)$ & 0.108 & & 0.037 \\
\hline \multicolumn{5}{|l|}{ BADL } \\
\hline Eating & & & 0.854 & 0.350 \\
\hline Dressing & & & 0.790 & 0.014 \\
\hline Taking a shower & & & 0.791 & 0.016 \\
\hline Using the toilet & & & 0.904 & 0.318 \\
\hline Moving from chair to bed and vice versa & & & 0.861 & 0.136 \\
\hline Incontinence & & & 1.063 & 0.513 \\
\hline \multicolumn{5}{|l|}{ IADL } \\
\hline Using the telephone & & & 1.007 & 0.944 \\
\hline Shopping & & & 0.916 & 0.330 \\
\hline Cooking & & & 0.866 & 0.129 \\
\hline Doing light housework & & & 0.884 & 0.178 \\
\hline Doing heavy housework & & & 0.925 & 0.419 \\
\hline Doing laundry & & & 0.830 & 0.042 \\
\hline Using public transport & & & 0.836 & 0.047 \\
\hline Managing medication & & & 0.860 & 0.104 \\
\hline Keeping track of finances & & & 0.868 & 0.135 \\
\hline Washing & & & 0.839 & 0.125 \\
\hline Walking upstairs & & & 0.864 & 0.120 \\
\hline Move from one room to another & & & 0.914 & 0.369 \\
\hline Walking on a flat floor & & & 0.880 & 0.195 \\
\hline
\end{tabular}

Table 3 Multiple linear and logistic regression model adjusted for age and sex showing the association of hemoglobin concentration with variables that describe the presence of comorbidities and creatinine and C-reactive protein levels

\begin{tabular}{|c|c|c|c|c|}
\hline $\begin{array}{l}\text { Hemoglobin concentra- } \\
\text { tion }(\mathrm{g} / \mathrm{dl}) \text { adjusted for } \\
\text { age, sex }\end{array}$ & $\beta \pm \mathrm{SE}(\beta)$ & $R^{2}$ & OR & $p$ \\
\hline Cardiovascular disease & & & 0.934 & 0.423 \\
\hline Cerebrovascular disease & & & 1.172 & 0.127 \\
\hline Myocardial infarction & & & 0.983 & 0.890 \\
\hline $\begin{array}{l}\text { Chronic pulmonary } \\
\text { disease }\end{array}$ & & & 0.845 & 0.136 \\
\hline $\begin{array}{l}\text { Peripheral vascular } \\
\text { disease }\end{array}$ & & & 0.875 & 0.216 \\
\hline Severe kidney disease & & & 0.572 & 0.005 \\
\hline Diabetes & & & 0.802 & 0.059 \\
\hline Cancer & & & 0.943 & 0.611 \\
\hline Ulcer & & & 0.848 & 0.137 \\
\hline Congestive heart failure & & & 0.818 & 0.045 \\
\hline Creatinine_tr & $-0.092(0.014)$ & 0.200 & & $<0.001$ \\
\hline C-reactive protein_tr & $-0.090(0.048)$ & 0.020 & & 0.064 \\
\hline
\end{tabular}

developing a disability in dressing and taking a shower activity decrease (Table 4 ).

\section{Discussion}

This study confirms that anemia is very common in the oldest old share of a community dwelling sample of Italian nonagenarians, with a significantly higher prevalence in men. Although hemoglobin concentration data is substantially identical in men $(n=74$; mean hemoglobin concentration $12.85+1.64)$ and women $(n=177$; mean hemoglobin concentration $12.84+1.43)$, the prevalence of anemia according to the WHO different cut offs for men and women are much higher in men (50\% of men vs $24.3 \%$ in women), with an overall $31.9 \%$ who fulfill the WHO criteria for anemia. These results are consistent with other reports on persons aged 85 and older (Leyden), and show a substantial increase of the anemic population compared to studies conducted on younger old patient samples [10, 20].

The appropriateness of the criteria for anemia set by the World Health Organization (WHO), i.e., hemoglobin 
Table 4 Multiple linear and logistic regression model adjusted for age, sex, comorbidities and creatinine level showing the association between hemoglobin concentration and variables that describe physical performance and disability

\begin{tabular}{|c|c|c|c|c|}
\hline $\begin{array}{l}\text { Hemoglobin concentration }(\mathrm{g} / \mathrm{dl}) \text { adjusted for } \\
\text { age, sex, comorbidities, creatinine }\end{array}$ & $\beta \pm \mathrm{SE}(\beta)$ & $\mathrm{R}^{2}$ & OR & $\mathrm{P}$ \\
\hline Handgrip_tr (kg) & $0.118(0.033)$ & 0.426 & & $<0.001$ \\
\hline Sps (score) & $0.348(0.169)$ & 0.111 & & 0.040 \\
\hline nADL (score) & $-0.484(0.210)$ & 0.182 & & 0.022 \\
\hline Score physical activity (score) & $0.094(0.037)$ & 0.137 & & 0.013 \\
\hline \multicolumn{5}{|l|}{ BADL } \\
\hline Dressing & & & 0.760 & 0.009 \\
\hline Taking a shower & & & 0.769 & 0.012 \\
\hline \multicolumn{5}{|l|}{ IADL } \\
\hline Doing laundry & & & 0.838 & 0.076 \\
\hline Using public transport & & & 0.847 & 0.092 \\
\hline
\end{tabular}

level $<12.00 \mathrm{~g} / 1$ for women and $<13.00 \mathrm{~g} / \mathrm{l}$ for men has been repeatedly questioned for older populations, as even hemoglobin just above the WHO anemia criteria is consistently associated with adverse outcome in older persons $[5,11,21]$. This is why in this study we chose to analyze the relationship of hemoglobin concentration with reduced physical performance, identified by two performance measures such as handgrip strength, which has been shown to be a proxy of muscle strength in the elderly [22], an early marker of poor mobility [19] and mortality in the oldest old [23] and with the Summary of Performance Score, whose lower scores are a powerful predictor of nursing home admission, hospitalization, and disability [24] while SPS higher scores are highly sensitive in discriminating those older persons who are not likely to experience adverse outcomes [25]. Indeed, SPPB might be used to assess frailty, by identifying subjects with a SPPB score of 9 and below. According to this cut-off value, most of our participants (77\%) are frail. We also verify the association of hemoglobin concentration with self-reported disability in basic and instrumental ADL and with self-reported physical activity, which in turn predicts subsequent disability even in the oldest old [26, 27]. Our results confirm a significant association between hemoglobin concentration and either Handgrip strength, SPS score, nADL score, as already reported elsewhere both for the younger and for the older old [10-12, 14]. The activities significantly associated with hemoglobin concentration in the regression analysis are dressing and taking a shower, which from a ADL hierarchy perspective correspond with a moderate stage disability [27].

So, as we confirm that even in the oldest old, low hemoglobin concentration is associated with self-reported disability and reduced physical performance, we then attempted to clarify the issue of whether this association is explained by underlying comorbidities that are highly prevalent in the oldest old. Since anemia and low hemoglobin concentration in old age are reported to be significantly associated and in large part etiologically related to chronic conditions such as cancer, renal failure, gastric ulcer and infection [5, 10], we first verified the association of hemoglobin concentration and these conditions in our study population. Indeed, we find that severe kidney disease and creatinine levels, as well as congestive heart failure are significantly associated with hemoglobin concentration, while we find no association with cancer, gastric ulcer and with the C-reactive protein, selected as a marker of inflammation/infection. This is a rather unexpected finding that may be at least partially explained by the high prevalence of these conditions in our study sample, $50 \%$ in men and $24 \%$ in women, compared to 11.1 and $11.5 \%$ observed in men and women, respectively, by Penninx et al. [10].

However, when we performed a multiple linear regression analysis including all the comorbid conditions associated with hemoglobin concentration as confounding factors, hemoglobin concentration level alone remains a powerful predictor of handgrip strength, and maintains a significant association with SPS, nADL and SPA scores. Thus, in contrast with what is reported in a large Dutch study on the 85-plus population, in our sample, low HC levels are associated with reduced self-reported physical activity, and are independently associated with reduced strength, physical performance, physical activity and disability.

The independent effect of hemoglobin concentration on physical functioning, as already reported in other relevant studies conducted on elderly population, may have several explanations. The impact on muscle strength may be related to a reduced muscle oxygenation [25]. Our results do not seem to confirm the hypothesis that the underlying cause both for anemia and for functional decline in the very elderly may be connected with chronic inflammation [10, 28], as we did not find a significant association between low HC levels and CRP. On the other hand, the pattern of disability associated with low hemoglobin concentration is different from that found in other age-associated conditions, such as musculoskeletal pain [29, 30], although reduced SPS scores are similarly found [31, 32]. 
Thus, the underlying causes leading to disability are probably related to specific mechanisms, such as for instance fatigue [10, 28], which may be increased by disuse: this possible mechanism may be confirmed in our sample by the significant cross-sectional association we find between reduced hemoglobin concentration and low self-reported physical activity.

The cross-sectional nature of our finding is the main limitation of this study; as the follow-up data are now being collected, a longitudinal analysis may shed light on the chronological and possibly etiological relationship between hemoglobin concentration and physical functioning. Further, although a comprehensive geriatric assessment and blood examinations were systematically performed, we did not thoroughly investigate the causes of anemia in our study population, as this would have exceeded the purpose of our study. So, it is possible that we may have missed the presence of subclinical conditions (such as cancer, gastric ulcer, bowel disease) that could have provided more insight on the relationship between hemoglobin concentration and physical function.

In sum, these findings confirm that anemia is highly prevalent in the oldest old. Further, our results confirm that low hemoglobin concentration is strongly associated with self-reported disability and reduced physical performance in the very elderly, in a way that is possibly independent of whatever is its clinical etiology. Anemia in the old population is multifactorial, and often goes undiagnosed, as approximately one third of anemia in the elderly remains of unknown origin [28]. Future research should provide more insight on the pathogenesis of anemia in this population [5, 28]. Further, as longitudinal research on nonagenarians is developing [33], the follow-up Mugello Study, as well as other studies should verify if low hemoglobin concentration in the very old will be a cause itself of adverse clinical and functional outcomes. If this will prove the case, the treatment of low hemoglobin concentration, even when the clinical etiology is not determinable or treatable in itself [34], may be considered as a mean to revert or reduce the risk of physical performance decline and of developing disability in the oldest old.

Acknowledgements This study was funded by Current Research Funds (2011/12) from the Italian Ministry of Health.

The Mugello Study Working Group also includes: Annamaria Gori, Nona Turcan, Debora Valecchi, Roberta Frandi, Lorenzo Razzolini, Valentina Fabbri, Guglielmo Bonaccorsi, Chiara Lorini.

\section{Compliance with ethical standards}

Conflict of interest The authors declare that they have no conflict of interest.

Statement of human and animal rights The study protocol, which complied with the principles of the Declaration of Helsinki on clinical research involving humans, was approved by Ethical Committee of the Don Gnocchi Foundation.

Informed consent Participants, or their legal representative, signed the informed consent form to be included in the study.

Open Access This article is distributed under the terms of the Creative Commons Attribution 4.0 International License (http://creativecommons.org/licenses/by/4.0/), which permits unrestricted use, distribution, and reproduction in any medium, provided you give appropriate credit to the original author(s) and the source, provide a link to the Creative Commons license, and indicate if changes were made.

\section{References}

1. World Health Organization, ageing and life course. http://www. who.int/ageing/en. Accessed Apr 2017

2. BC News Service (2007) http://news.bbc.3.co.uk/1/hi/magazine/7016844.stm. Accessed Apr 2017

3. Balducci L (2003) Epidemiology of anemia in the elderly: information on diagnostic evaluation. J Am Geriatr Soc 51:S2-S9

4. Zafrir B, Laor A, Bitterman H (2010) Nonagenarians in internal medicine: characteristics, outcomes and predictors for in-hospital and post-discharge mortality. Isr Med Assoc J 12(1):10-15

5. Beghé C, Wilson A, Ershler WB (2004) Prevalence and outcomes of anemia in geriatrics: a systematic review of the literature. Am J Med 5:116

6. Zakai NA, Katz R, Hirsch C et al (2005) A prospective study of anemia status, hemoglobin concentration, and mortality in an elderly cohort: the Cardiovascular Health Study. Arch Intern Med 165(19):2214-2220

7. Patel KV, Harris TB, Faulhaber M et al (2007) Racial variation in the relationship of anemia with mortality and mobility disability among older adults. Blood 109(11):4663-4670

8. Joosten E, Detroyer E, Milisen K (2016) Effect of anaemia on hand grip strength, walking speed, functionality and 1 year mortality in older hospitalized patients. BMC Geriatr 16(1):153

9. Dharmarajan TS, Avula S, Norkus EP (2006) Anemia increases risk for falls in hospitalized older adults: an evaluation of falls in 362 hospitalized, ambulatory, long-term care, and community patients. J Am Med Dir Assoc 7(5):287-293

10. Penninx BW, Pahor M, Cesari M, Corsi AM, Woodman RC, Bandinelli S, Guralnik JM, Ferrucci L (2004) Anemia is associated with disability and decreased physical performance and muscle strength in the elderly. J Am Geriatr Soc 52(5):719-724

11. Pires Corona L, Drumond Andrade FC, de Oliveira Duarte YA, Lebrao ML (2014) The relationship between anemia, hemoglobin concentration and frailty in Brazilian older adults. J Nutr Health Aging 3:336-340

12. Juárez-Cedillo T, Basurto-Acevedo L, Vega-García S, ManuelApolinar L, Cruz-Tesoro E, Rodríguez-Pérez JM, GarcíaHernández N, Pérez-Hernández N, Fragoso JM (2014) Prevalence of anemia and its impact on the state of frailty in elderly people living in the community: SADEM study. Ann Hematol 93(12):2057-2062

13. Röhrig G, Becker I, Schulz RJ, Lenzen-Großimlinghaus R, Willschrei P, Gebauer S, Modreker M, Jäger M, Wirth R (2016) Association between hematologic parameters and functional impairment among geriatric inpatients: data of a prospective cross-sectional multicenter study ("GeriPrävalenz2013"). Maturitas 90:37-41

14. den Elzen WP, Willems JM, Westendorp RG, de Craen AJ, Assendelft WJ, Gussekloo J (2009) Effect of anemia and 
comorbidity on functional status and mortality in old age: results from the Leiden 85-plus study. CMAJ 181(3-4):151-157

15. Molino-Lova R, Sofi F, Pasquini G, Gori A, Vannetti F, Abbate R, Gensini GF, Macchi C (2013) The Mugello Study, a survey of nonagenarians living in Tuscany: design, methods and participants' general characteristics. Eur J Intern Med 24(8):745-749

16. Katz S, Ford A, Moskowitz R et al (1963) Studies of illness in the aged: index of ADL. A standardized measure of biological and psychological function. JAMA 185:94-99

17. Lawton MP, Brody EM (1969) Assessment of older people: self-maintaining and instrumental activities of daily living. Gerontologist 9:179-186

18. Guralnik JM, Simonsick EM, Ferrucci L et al (1994) A short physical performance battery assessing lower extremity function: association with self-reported disability and prediction of mortality and nursing home admission. J Gerontol 49:M85-M94

19. Lauretani F, Russo CR, Bandinelli S, Bartali B, Cavazzini C, Di Iorio A, Corsi AM, Rantanen T, Guralnik JM, Ferrucci L (2003) Age-associated changes in skeletal muscles and their effect on mobility: an operational diagnosis of sarcopenia. J Appl Physiol (1985) 95(5):1851-1860

20. Guralnik JM, Eisenstaedt RS, Ferrucci L, Klein HG, Woodman RC (2004) Prevalence of anemia in persons 65 years and older in the United States: evidence for a high rate of unexplained anemia. Blood 104(8):2263-2268

21. Chaves PH, Ashar B, Guralnik JM et al (2002) Looking at the relationship between hemoglobin concentration and prevalent mobility difficulty in older women. Should the criteria currently used to define anemia in older people be reevaluated. J Am Geriatr Soc 50:1257-1264

22. Ferrucci L, Bandinelli S, Benvenuti E et al (2000) Subsystems contributing to the decline in ability to walk: bridging the gap between epidemiology and geriatric practice in the InCHIANTI study. J Am Geriatr Soc 48:1618-1625

23. Ling CHY, Taekema D, de Craen AJM, Gussekloo J, Westendorp RGJ, Maier AB (2010) Handgrip strength and mortality in the oldest old population: the Leiden 85-plus study. CMAJ: Can Med Assoc J 182(5):429-435

24. Guralnik JM, Ferrucci L, Simonsick EM et al (1995) Lowerextremity function in persons over the age of 70 years as a predictor of subsequent disability. N Engl J Med 332:556-561
25. Pedone C, Costanzo L, Cesari M, Bandinelli S, Ferrucci L, Antonelli Incalzi R (2016) Are performance measures necessary to predict loss of independence in elderly people? J Gerontol A Biol Sci Med Sci 71(1):84-89

26. den Ouden ME, Schuurmans MJ, Arts IE, van der Schouw YT (2011) Physical performance characteristics related to disability in older persons: a systematic review. Maturitas 69(3):208-219

27. Vermeulen J, Neyens JC, van Rossum E, Spreeuwenberg MD, de Witte LP (2011) Predicting ADL disability in community-dwelling elderly people using physical frailty indicators: a systematic review. BMC Geriatr 1(11):33. doi:10.1186/1471-2318-11-33

28. Andrès E, Serraj K, Federici L, Vogel T, Kaltenbach G (2013) Anemia in elderly patients: new insight into an old disorder. Geriatr Gerontol Int 13(3):519-527

29. Cecchi F, Mannoni A, Molino-Lova R, Ceppatelli S, Benvenuti E, Bandinelli S, Lauretani F, Macchi C, Ferrucci L (2008) Epidemiology of hip and knee pain in a community based sample of Italian persons aged 65 and older. Osteoarthr Cartil 16(9):1039-1046

30. Cecchi F, Debolini P, Lova RM, Macchi C, Bandinelli S, Bartali B, Lauretani F, Benvenuti E, Hicks G, Ferrucci L (2006) Epidemiology of back pain in a representative cohort of Italian persons 65 years of age and older: the InCHIANTI study. Spine 31(10):1149-1155

31. Cecchi F, Molino-Lova R, Di Iorio A, Conti AA, Mannoni A, Lauretani F, Benvenuti E, Bandinelli S, Macchi C, Ferrucci L (2009) Measures of physical performance capture the excess disability associated with hip pain or knee pain in older persons. J Gerontol A Biol Sci Med Sci 64(12):1316-1324

32. Di Iorio A, Abate M, Guralnik JM, Bandinelli S, Cecchi F, Cherubini A, Corsonello A, Foschini A, Guglielmi M, Lauretani F, Volpato S, Abate G, Ferrucci L (2007) From chronic low back pain to disability, a multifactorial mediated pathway: the InCHIANTI study. Spine 32(26):E809-E815

33. Formiga F, Ferrer A, Espaulella J, Rodriguez-Molinero A, Chivite D, Pujol R (2010) Decline in the performance of activities of daily living over three years of follow-up in nonagenarians: the NonaSantfeliu study. Eur Geriatr Med 1(2):77-81 (ISSN 1878-7649)

34. Thomas DR (2007) Anemia: it's all about quality of life. J Am Med Dir Assoc 8:80-82. doi:10.1016/j.jamda.2006.12.025 